\title{
Melatonin protects podocytes from angiotensin II-induced injury in an in vitro diabetic nephropathy model
}

\author{
ZHEN-ZHONG JI ${ }^{1}$ and YAN-CHENG XU ${ }^{2}$ \\ Departments of ${ }^{1}$ Integrated Wards and ${ }^{2}$ Endocrinology, Zhongnan Hospital of Wuhan University, \\ Wuhan, Hubei 430071, P.R. China
}

Received July 8, 2015; Accepted May 6, 2016

DOI: $10.3892 / \mathrm{mmr} .2016 .5313$

\begin{abstract}
Diabetic nephropathy (DN) is a severe renal disorder characterized by podocyte damage and accumulation of extracellular matrix leading to further glomerulosclerosis, possibly via the activation of inflammatory signaling and the generation of oxidative stress. Melatonin has been considered a robust anti-oxidant, and is able to attenuate DN in several animal models. In the present study, cell viability was examined by CCK-8 assay. Cell apoptosis, intracellular reactive oxygen species and mitochondrial membrane potential (MMP) levels were measured by flow cytometry. Western blot analysis was performed to detect the expression of Nephrin, B cell lymphoma-2 (Bcl-2)-associated X protein (Bax), Bcl-2, Caspase-3, Janus kinase (JAK)2 and Signal transducer and activator of transcription (STAT) 3 in podocytes. Based on an in vitro podocyte injury model, the present study found that the application of melatonin significantly reduced AngII-induced apoptosis and increased the proliferative rate of cells, as evidenced by decreased expression levels of apoptotic proteins, including Caspase- 3 and Bax, and a change in the ratio of $\mathrm{Bax} / \mathrm{Bcl}-2$. Further investigation demonstrated that a reduction in oxidative stress and the recovery of mitochondrial function were involved in this protective effect. In addition, the Jak/STAT signaling pathway was inhibited, indicating that cytokine-mediated inflammation was also targeted by melatonin. The present study demonstrated for the first time, to the best our knowledge, that melatonin exerted an anti-apoptotic effect in AngII-mediated podocyte injury, and indicates a possible mechanism to explain the protective effect of melatonin in DN.
\end{abstract}

Correspondence to: Professor Yan-Cheng $\mathrm{Xu}$, Department of Endocrinology, Zhongnan Hospital of Wuhan University, 169 Donghu Road, Wuchang, Wuhan, Hubei 430071, P.R. China E-mail: xuyancheng_p@126.com

Key words: diabetic nephropathy, podocytes, angiotensin II, melatonin, oxidative stress, antioxidant

\section{Introduction}

Diabetic nephropathy (DN) is the leading cause of end-stage renal disease in the US, which is characterized by damage to podocytes and increased extracellular matrix accumulation $(1,2)$, which leads to the progression of nodular glomerulosclerosis, micro-albuminuria and eventual renal failure. The molecular and cellular mechanisms responsible for this disease remain to be fully elucidated. Previous clinical and experimental studies have demonstrated that the local growth factor, angiotensin II (AngII) requires consideration as a vital mediator in the pathogenesis in DN (3). The inhibition of the renin-angiotensin system by angiotensin-converting enzyme inhibitors ameliorates proteinuria and the progression of nephropathy, which suggest that this inhibition may have beneficial effects on various types of renal cell (4-6).

Podocyte injury is the hallmark characteristic of DN (7). The local production of AngII in podocytes contributes to the development of kidney disease through the generation of reactive oxygen species (ROS) and oxidative stress, resulting in podocyte apoptosis, matrix accumulation and aberrant proliferation (8-10). Several studies have reported that, during the progression of DN, the suppression of superoxide generation in response to AngII attenuates renal function, although results following the application of antioxidants remain inconsistent $(11,12)$. Several potentially beneficial actions of antioxidant administration on DN have been reported in human and experimental diabetes (12). The present study aimed to examine whether the antioxidant melatonin can ameliorate pathophysiological injury in podocytes, including severe oxidative stress and apoptosis, in an in vitro AngII-induced DN model.

Melatonin is the predominant hormone secreted by the pineal gland and is involved in sustaining circadian rhythms in various biological processes. Several biological effects of melatonin are mediated through the activation of melatonin receptors, whereas others are a result of its powerful anti-oxidative function. Several studies have found that melatonin prevents renal damage in Type I and Type II diabetes via reducing free radical attack (13-16), however, the cell-specific mechanism remains to be fully elucidated.

The present study demonstrated for the first time to the best our knowledge, that melatonin exerts an anti-apoptotic effect 
in AngII-mediated podocyte injury, and indicates a possible mechanism to explain the protective effect of melatonin in DN.

\section{Materials and methods}

Reagents and cell line. Angiotensin II (A9290-10) was purchased from Solarbio Bioscience \& Technology, Inc. (Shanghai, China). The chemical reagents used for detecting cell proliferation and apoptosis comprised a Cell Counting kit-8 (CCK-8; cat. no. CK04; Dojindo Molecular Technologies, Inc. Kumamoto, Japan) and an Annexin V-Fluorescein isothiocyanate (FITC) apoptosis detection kit (BD Pharmingen, San Diego, CA, USA; cat. no. 556547). A reactive oxygen species (ROS) assay kit (Vigorous Biotech, Beijing, China) and MitoPT $^{\mathrm{TM}}$ JC-1 assay kit (ImmunoChemistry Technologies, LLC, Bloomington, MN, USA; cat. no. 924) were used to investigate mitochondrial function.

Cell culture and treatment. Mouse podocytes $\left(5 \times 10^{4}\right.$ cells $\left./ \mathrm{ml}\right)$ were acquired from JRDun Biotechnology Co., Ltd. (Shanghai, China) and propagated at $33^{\circ} \mathrm{C}$ in RPMI-1640 culture media supplemented with $10 \%$ fetal bovine serum (FBS; GE Healthcare Life Sciences HyClone Laboratories, Logan, UT, USE) and $1,000 \mathrm{U} / \mathrm{ml}$ recombinant interferon (BioVision, Inc., Milpitas, CA, USA) to enhance the expression of a thermo-sensitive $\mathrm{T}$ antigen, as previously described (17). To establish an injury model, the podocytes were seeded at $80 \%$ confluence in complete medium containing 10\% FBS. After $24 \mathrm{~h}$, the cell culture medium was replaced with serum-free medium containing $5 \mu \mathrm{mol}$ Ang II for $24 \mathrm{~h}$ at $37^{\circ} \mathrm{C}$. Subsequently, different concentrations of melatonin (Shanghai Amquar Biological Technology Co., Ltd., Shanghai, China) were added for various periods of time, as indicated. The cells were collected at different time points for further characterization.

Apoptosis assay. The cells were washed, trypsinized and suspended in binding buffer, and the density was adjusted to $5-10 \times 10^{5} / \mathrm{ml}$. Apoptosis was measured by staining with Annexin V- FITC and propidium iodide (PI). The podocytes in suspension (195 $\mu \mathrm{l}$ ) was added to $5 \mu \mathrm{l}$ Annexin V-FITC and $10 \mu 1$ PI. The stained cells were analyzed by fluorescence-activated cell-sorting (FACS; FACScalibur; Becton Dickinson, Mountain View, CA, USA). The apoptotic cells were calculated as the percentage of IV-gated cells.

Cell viability assay. The podocytes $\left(1 \times 10^{3}\right.$ per well) were plated in 96-well plates. Following treatment with Ang II for $24 \mathrm{~h}$, a series of melatonin concentrations $(0.1,0.5$ and $1.0 \mathrm{mmol})$ were added for different durations (48 and $72 \mathrm{~h}$ ). Subsequently, 10\% CCK-8 diluted in serum-free RPMI-1640 (Invitrogen, Thermo Fisher Scientific, Inc., Waltham, MA, USA) was added to each well for $1 \mathrm{~h}$ at $37^{\circ} \mathrm{C}$. The absorption of each sample was measured at a wavelength of $450 \mathrm{~nm}$ using a Labsystems MK3 microplate reader (Thermo Fisher Scientific, Inc.) to detect cell viability, according to the manufacturer's protocol. Cells without Ang II treatment served as a control group. Triplicate experiments were performed throughout all the procedures.

Measurement of intracellular ROS levels. The levels of intracellular ROS were measured using a ROS assay kit, according to the manufacturer's protocol. Briefly, the treated podocytes were washed three times with pre-warmed phosphate-buffered saline (PBS) and then harvested for further staining. Dihydroethidium (DHE; $50 \mu \mathrm{M}$ ) was added to the cells for incubation at room temperature for $30 \mathrm{~min}$, following which the cells were analyzed using FACS. The fluorescence intensity was monitored at an excitation wavelength of $488 \mathrm{~nm}$ and an emission wavelength of $605 \mathrm{~nm}$.

Detection of mitochondrial membrane potential (MMP). The treated podocytes were washed with pre-warmed PBS, trypsinized, centrifuged ( $400 \mathrm{x} \mathrm{g}$ at room temperature) for $10 \mathrm{~min}$ and adjusted to $1 \times 10^{6} \mathrm{cell} / \mathrm{ml}$. Tetramethylrhodamine, methyl ester (TMRM) was used as a trans-membrane dye to indicate the function of the mitochondria. The cells were incubated with $100 \mathrm{nM}$ TMRM medium at $37^{\circ} \mathrm{C}$ for $15-20 \mathrm{~min}$ in the dark, and washed again with PBS to remove any unbound probe. Analysis was performed using FACS at an excitation wavelength of $543 \mathrm{~nm}$ and an emission wavelength of $589 \mathrm{~nm}$.

Western blot analysis. The podocytes were washed with PBS several times and then added to lysis buffer premixed with proteinase and phosphatase inhibitors. The concentration of protein was quantified using a bicinchoninic acid assay method (Thermo Fisher Scientific, Inc.). Subsequently, $35 \mu \mathrm{g}$ protein for each sample was loaded onto $12 \%$ SDS-PAGE gels for separation, and further transferred onto a polyvinylidene fluoride membrane. The blots were blocked with 5\% non-fat milk and incubated overnight with the following primary antibodies: Rabbit anti-nephrine (Abcam, Cambridge, UK; cat. no. Ab58968; 1:1,000 dilution); rabbit anti-B cell lymphoma-2 (Bcl-2; Santa Cruz Biotechnology, Inc., Santa Cruz, CA, USA; cat. no. SC-492; 1:150 dilution); rabbit anti-Bcl-2-associated X protein (Bax; Santa Cruz Biotechnology, Inc.; cat. no. sc-493; 1:100 dilution); rabbit anti-caspase-3 (Abcam; cat. no. Ab32351; 1:2,000 dilution); rabbit anti-phosphorylated (phospho)-Janus kinase (JAK)2 (Cell Signaling Technology, Inc., Danvers, MA, USA; cat. no. 3776; 1:800 dilution); rabbit anti-JAK2 (Cell Signaling Technology, Inc.; cat. no. 3230; 1:1,000 dilution); rabbit anti-phospho-Signal transducer and activator of transcription 3 (STAT3; Abcam; cat. no. Ab76315; 1:800 dilution); mouse anti-STAT3 (Abcam; cat. no. Ab119352; 1:1,000 dilution); rabbit anti-GAPDH (Cell Signaling Technology, Inc.; cat. no. 5174; 1:1,500 dilution). GAPDH was used for the normalization of each protein to ensure the loading of equal quantities of protein. Following washing three times with Tris-buffered saline-tween buffer, the blots were incubated for $1 \mathrm{~h}$ at room temperature with goat anti-rabbit and goat anti-mouse horseradish peroxidase-conjugated secondary antibodies (cat. nos. A0208 and A0216, respectively; 1:1,000; Beyotime Institute of Biotechnology, Haimen, China). Following another round of washing, the signals were revealed using enhanced chemiluminescence (EMD Millipore, Billerica, MA, USA).

Statistical analysis. Statistical analyses were performed using GraphPad Prism 5 software (GraphPad Software, Inc., La Jolla, CA, USA). Statistical analysis was performed using one-way analysis of variance tests. $\mathrm{P}<0.05$ was considered to indicate a statistically significant difference. Data are 
expressed as the mean \pm standard deviation of triplicate samples. All results were confirmed in at least three independent experiments.

\section{Results}

Melatonin protects podocytes from Ang II-induced apoptosis. In order to investigate the function of melatonin in DN, the present study established an in vitro model, in which Ang II was administered to podocytes. Following treatment with Ang II for $24 \mathrm{~h}$, the podocytes were stained with Annexin V for further analysis. The FACS results showed the injured podocytes exhibited high levels of apoptosis $(45.800 \pm 0.929 \%)$, compared with the control group $(0.103 \pm 0.003 \%$; Fig. 1). Following exposure to different dosages of melatonin for another $48 \mathrm{~h}$, the apoptotic rates of the podocytes were significantly decreased, between $36.87 \%$ ( $0.1 \mathrm{mM}$ melatonin) and $17.70 \%$ (1 mM melatonin). These results indicated that melatonin protected the podocytes from Ang II-induced apoptosis in a dose-dependent manner.

Melatonin restores cell viability in an Ang II-induced podocytes injury model. The results of the CCK-8 assay demonstrated that, following $12 \mathrm{~h}$ Ang II treatment, the viability of the podocytes was reduced between $0.334 \pm 0.005$ and $0.285 \pm 0.005$, and continued to decrease significantly, between $0.832 \pm 0.005$ and $0.476 \pm 0.006$, until 72 h (Fig. 2). However, melatonin ameliorated this damage and restored cell viability in a concentration-dependent manner (Fig. 2). Following incubation for $12 \mathrm{~h}$, the high concentration of melatonin $(1 \mathrm{mmol})$ rescued almost all the podocytes from injury ( $1 \mathrm{mmol}$ melatonin, $0.319 \pm 0.002$; control group, $0.334 \pm 0.005$ ). As the duration of incubation increased, this protective effect was reduced, with $>50 \%$ of injured podocytes rescued post-injury following $72 \mathrm{~h}$ in high-concentration melatonin (1 mmol melatonin, 0.622 \pm 0.006 ; control group, $0.832 \pm 0.005$ ).

Effect of melatonin on mitochondrial function and intracellular ROS in injured podocytes. Melatonin has been confirmed as a powerful anti-oxidant. In order to address the underlying mechanism in the present study, the mitochondrial function and production of ROS in the injured podocytes were measured. The use of fluorescent dye for the assessment of MMP has become a useful tool for monitoring changes in physiological parameters, as it associated with the capacity of the cell to generate ATP and maintain normal mitochondria function. Treatment with Ang II for $24 \mathrm{~h}$ markedly decreased MMP, compared with the control group ( $0.38 \%$ of the control group (Fig. 3 ) and reduced mitochondrial function. Incubation for $48 \mathrm{~h}$ with a low concentration of melatonin $(0.1 \mathrm{mmol})$ was able to partially repair MMP, and a high concentration melatonin restored almost $40 \%$ of the mitochondrial function, compared withe the control group (Fig. 3). Concomitantly, intracellular ROS was produced due to the damage of MMP, which further reduced mitochondrial function. As shown in Fig. 4, the results confirmed that Ang II induced the production of ROS, with an increase of up to 360 -fold, compared with the normal podocytes. Treatment with melatonin exhibited its potent anti-oxidant effect and eliminated ROS in a dose-dependent manner. In the cells incubated with a high concentration of melatonin, ROS were cleared to almost the same level as in the normal group. Taken together, these results confirmed melatonin as a potent anti-oxidative reagent, and demonstrated that melatonin attenuated damage to the podocytes via the clearance of ROS.

Effects of melatonin on apoptosis and the JAK/STAT signaling pathway. As described above, melatonin exerted a protective effect on the podocytes against apoptosis and increased cell viability. In addition, certain molecules associated with apoptosis and cell proliferation signaling pathways were detected. Nephrin is a major cell membrane component connecting the cytoskeleton in podocytes and is lost during podocyte injury. As shown in Fig. 5, Ang II decreased the expression of Nephrin (lane B) and melatonin restored its expression, which increased in a concentration-dependent manner (Lanes C-E). The Bax/Bcl-2 families are known to be important in the regulation of apoptosis, and the increased ratio of $\mathrm{Bax} / \mathrm{Bcl}-2$ can trigger Caspase activation and downstream apoptosis. In the present study, Ang II increased the expression of Bax and repressed the expression of $\mathrm{Bcl}-2$ level, this change in the $\mathrm{Bax} / \mathrm{Bcl}-2$ ratio was corrected by melatonin. Dose-dependent changes in the expression levels of Bax and Bcl-2 were observed in the melatonin-treated podocytes. Caspase- 3 activation was also suppressed by melatonin.

The STAT family of transcription factors can control the activation of several signaling pathways, including inflammation, proliferation and apoptosis. STAT transcription factors are activated by tyrosine phosphorylation, predominantly JAK. In the present study, activation of JAK2/STAT3 phosphorylation was observed in response to AngII-mediated oxidative stress and inflammatory disorder. Again, melatonin exerted its anti-oxidative and anti-inflammatory effects, and the activation of the JAK/STAT pathway was reduced by melatonin in a dose-dependent manner.

\section{Discussion}

DN is one of the predominant causes of end-stage renal disease. It affects $\sim 30 \%$ of patients with Type I diabetes, and $20 \%$ of patients with diabetes $<40$ years of age succumb to DN-associated mortality (18). The glomerulus is the most commonly affected structure in DN, and is characterized by disordered synthesis of the mesangial matrix (19), loss of functional cytoskeleton connections (20) and enhanced apoptotic rate in podocytes during the progression of diabetes $(21,22)$, which is partly mediated by Ang II. Earlier studies have indicated that, as a potent anti-oxidant, chronic administration of melatonin showed potent beneficial effects in ameliorating DN in the rat renal tubule and glomerulus $(13,23)$, and this may have been via a reduction in oxidative stress. However, few investigations have focused on elucidating the intracellular mechanisms of the protective function of melatonin on specific cell types in the kidney. In the present study, experiments aimed to elucidate the possible mechanism in podocytes by which melatonin protects the kidney from oxidative stress and renal damage.

Ang II is considered to be a vital mediator in the progression of DN (24), as demonstrated in the results of the present study, in which $24 \mathrm{~h}$ incubation with Ang IIl led to marked podocyte damage. As shown in Fig. 1, the podocytes were sensitive to Ang II-induced apoptosis. A study by Gumustekin et al (25) demonstrated the presence of TUNEL-positive cells in the 

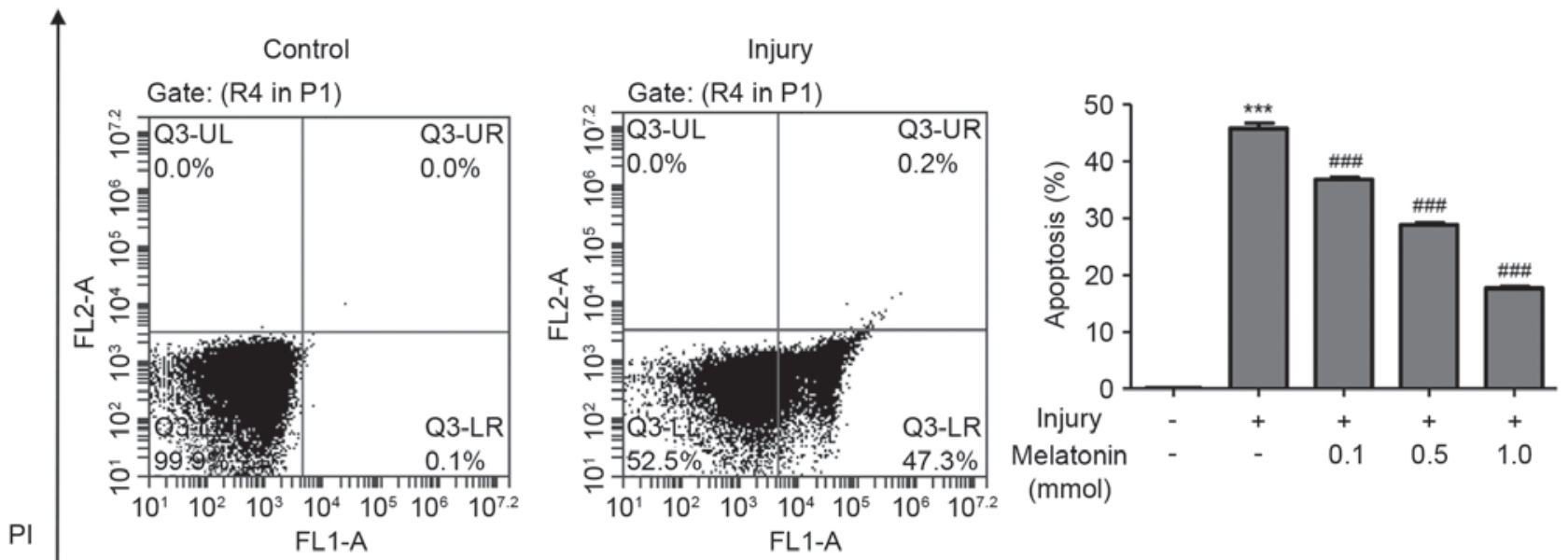

Injury+0.1 mmol melatonin
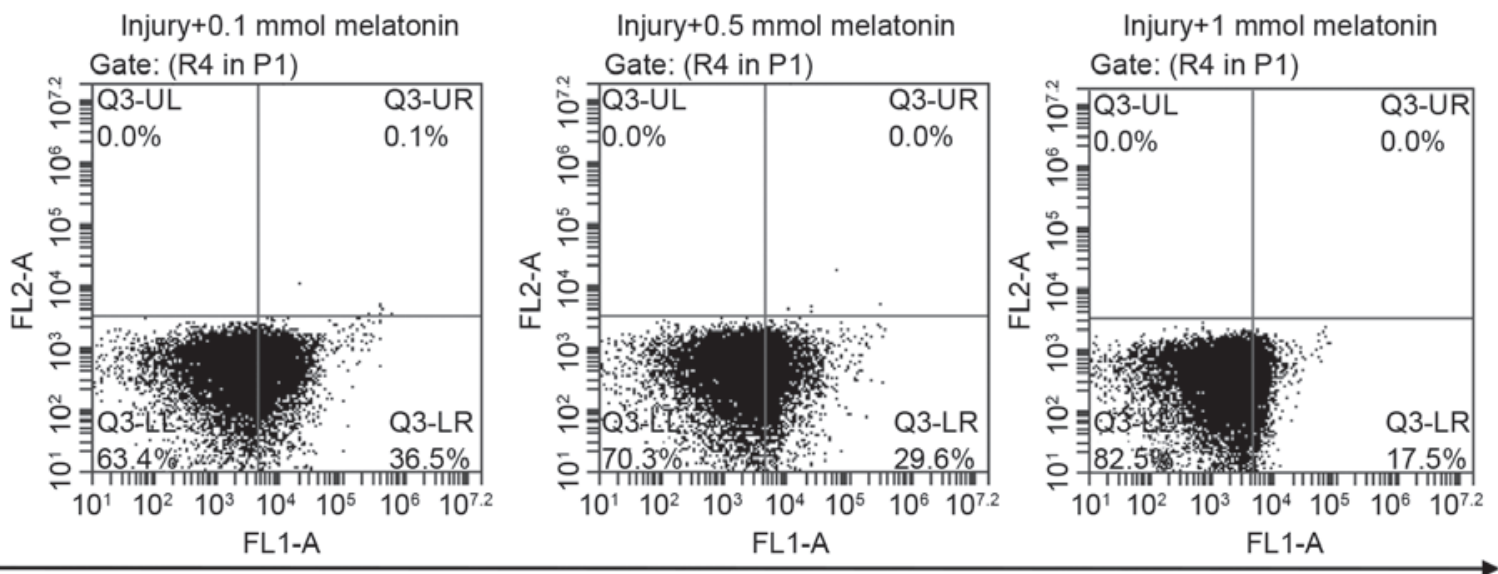

Annexin V

Figure 1. Percentages of apoptotic podocytes, measured using Annexin V-fluorescein isothiocyanate and PI staining. IV gated cells were indicative of apoptosis. Melatonin significantly decreased the percentage of apoptotic podocytes induced by Ang II. Data are expressed as the mean \pm standard deviation. ${ }^{* * * *} \mathrm{P}<0.001$, injury group, vs. control group; ${ }^{\# \# /} \mathrm{P}<0.001$, melatonin group, vs. injury group. Ang II, angiotensin II; PI, propidium iodide.

glomerular mesangium and tubular epithelial cells in diabetic rat, and observed that treatment with melatonin effectively reduced its expression. Similarly, melatonin-treated podocytes were protected from Ang II-induced apoptosis in the present study, as shown in Fig. 1. In addition to offering protection from apoptosis, melatonin increased the number of living cells and rescued the cells from Ang II-induced cell death (Fig. 2). At a high concentration, almost $50 \%$ of the podocytes were rescued. Taken together, these results confirmed the beneficial effect of melatonin in podocytes.

MMP is an important physiological parameter as it is associated with the capacity of the cell to generate ATP and control energy homeostasis $(26,27)$. During cellular stress, MMP may be adversely altered by the dysfunction of intracellular ionic charges, consequently leading to the failure of ATP production and the generation of ROS (27). Therefore, MMP is a key indicator of cell death and injury. As demonstrated in the present study, Ang II caused a marked decrease in the MMP of the podocytes (Fig. 3), indicating the possibility of mitochondrial injury. Several previous studies have reported that, during the progression of DN in patients and animal models, oxidative stress is considered a crucial risk factor contributing to the pathogenesis of renal disease (28-30). The occurrence of high glucose levels (31) and exposure to mechanical stress (22) in DN may induce the local expression of Ang II, acting through

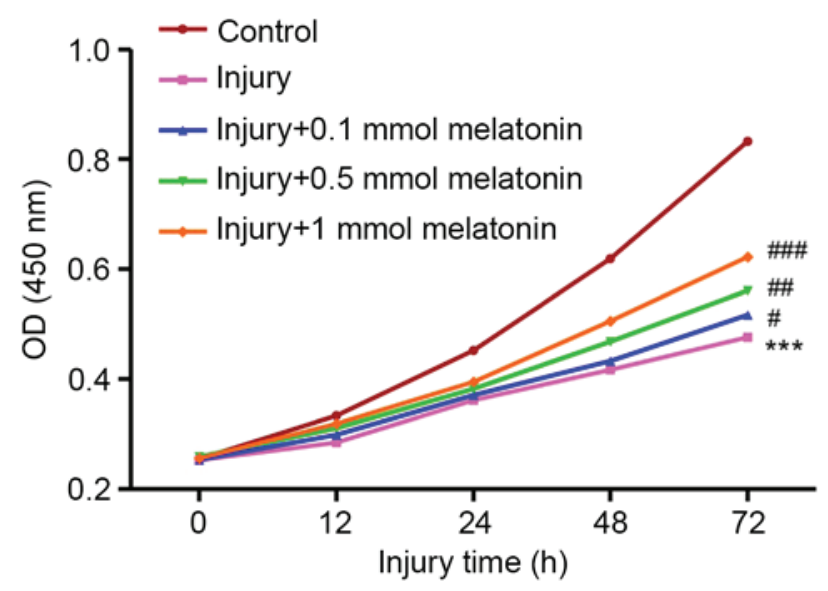

Figure 2. Assessment of podocyte viability using a Cell Counting kit- 8 assay. Dose-dependent effects of melatonin were observed on the viability of podocytes injured by Ang II treatment for $24 \mathrm{~h}$. At the late stage of injury, melatonin significantly ameliorated Ang II-induced cell loss. ${ }^{* * * *} \mathrm{P}<0.001$, injury group, vs. control group; ${ }^{\#} \mathrm{P}<0.05$, ${ }^{\# \#} \mathrm{P}<0.01$ and ${ }^{\# \# \#} \mathrm{P}<0.001$, melatonin group, vs. injury group at different time points. Ang II, angiotensin II; OD, optical density.

Angiotensin II receptors, which aggravate podocyte injury. As shown in Fig. 4, Ang II not only damaged MMP, it also produced life-threatening levels of ROS simultaneously. Melatonin has 


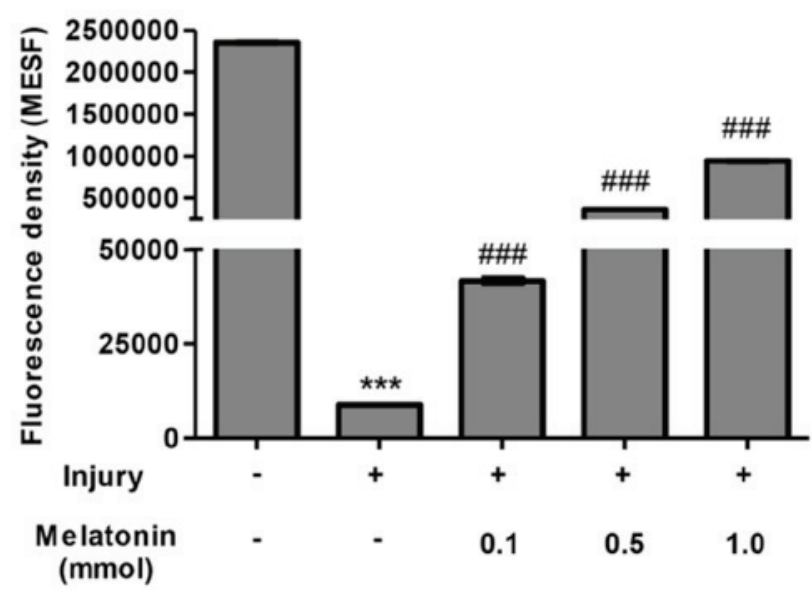

Figure 3. Measurement of MMP in podocytes. The fluorescent dye, tetramethylrhodamine, methyl ester was used to examine the mitochondria. Melatonin treatment rescued the Ang II-mediated decrease in MMP in a dose-dependent manner. Data are expressed as the mean \pm standard deviation. ${ }^{* * *} \mathrm{P}<0.001$, injury group, vs. Control group; ${ }^{\# \#} \mathrm{P}<0.001$, melatonin group, vs. injury group. Ang II, angiotensin II; MMP, mitochondrial membrane potential.

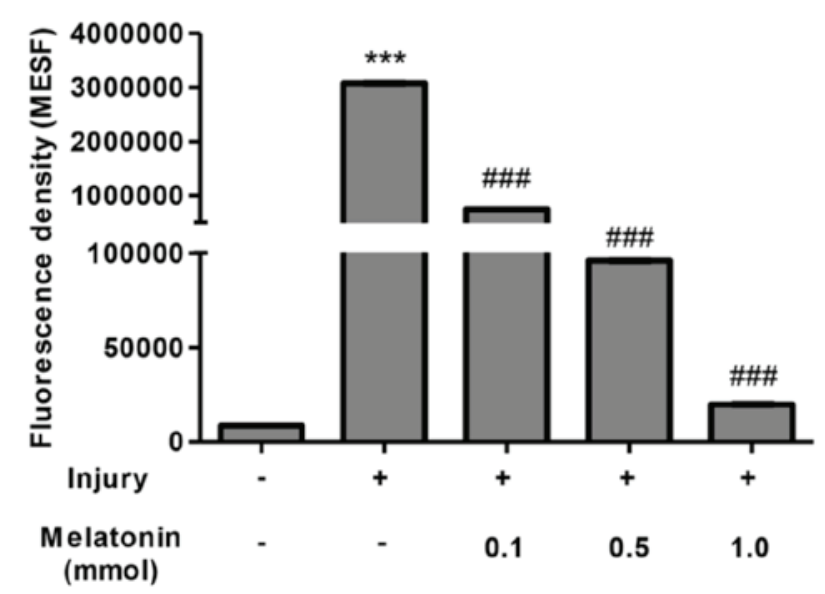

Figure 4. Detection of intracellular ROS using the DHE probe. The treated podocytes were stained with DHE for analysis using a fluorescence-activated cell-sorting machine. Angiotensin II induced the production of ROS, and this was inhibited by melatonin in a dose-dependent manner. Data are expressed as the mean \pm standard deviation. ${ }^{* * *} \mathrm{P}<0.001$, injury group vs. control group; ${ }^{\# \# \#} \mathrm{P}<0.001$, melatonin group vs. injury group. ROS, reactive oxygen species; DHE, dihydroethidium.

been confirmed as a potent anti-oxidant. As early as 1999, Ha et al demonstrated that water supplemented with melatonin reduced the accumulation of plasma lipid peroxides in streptozotocin-induced DN in rats (13). In subsequent investigations, it was suggested that melatonin may exert its renal protective effects in several aspects, for example the clearance of superoxide species in plasma or urine, including lipid peroxides (13); and increasing the activities of superoxide dismutase, xanthine oxidase, glutathione peroxidase and ceruloplasmin $(14,16,23)$. Although several studies have shown discrepancy in the function of melatonin in the activity of catalase and superoxide dismutase, there is substantial evidence supporting the anti-oxidative protection offered by melatonin and in podocytes, the results of the present study demonstrated that different concentrations of melatonin cleared the Ang II-induced production of ROS and rescued damaged MMP. Taken together, the present study found that melatonin provided protection in DN through preventing podocyte exposure to oxidative stress-induced damage.

Podocytes form slit pores, through which the glomerular filtrate passes, and alterations in this structure are responsible for the leakage of proteins and blood cells observed in DN (32). Nephrin is the predominant component in the family of podocyte membrane proteins, and is present with other members connected to the actin cytoskeleton to maintain the normal structure of the glomerulus (33). In the present study, although Ang II decreased the expression of nephrin, the application of melatonin prevented its suppression in a dose-dependent manner, indicating recovery of the glomerular cytoskeleton and general structure.

Mitochondrial outer membrane permeabilization is associated with mitochondrial dysfunction or MMP, and is responsible for the release of soluble proteins, which are closely associated with cell death (34). Once homeostasis is impaired, a mitochondrial-associated cell death pathway is immediately activated. The release of cytochrome $c$ into the cytoplasm mediates the binding of apoptotic protease activating factor-1 to pro-caspase-9 (34), resulting in the activation of caspase-9 and initiation of the caspase cascade, including caspase 3, and DNA damage. The immediate cellular response to DNA damage includes cleavage of poly ADP ribose polymerase by caspase- 3 . However, the release of cytochrome $c$ is tightly controlled by members of the Bcl-2 family, known to be associated with the regulation of apoptosis $(34,35)$. An increase in the level of Bax or decrease in the level of Bcl-2 can shift the ratio and trigger the apoptotic signal. In the podocytes examined in the present study, incubation with AngII activated the expression of caspase-3 and altered the ratio of Bax/Bcl-2 (lane B, vs. A; Fig. 5), which led to apoptosis in the Ang II group (Fig. 1). However, in the present study, dose-dependent downregulation in the expression of caspase 3 and changes in the $\mathrm{Bax} / \mathrm{Bcl}-2$ ratio were observed in the melatonin-treated podocytes, confirming its protective role as an anti-oxidant.

STAT proteins belong to a superfamily controlling transcription-regulating signaling and the response of cells to environmental stimuli in the absence of protein synthesis through the STAT pathway (36). In mammals, there are seven STAT genes, and STAT3 has been widely investigated due to its numerous functions, including animal cell growth regulation and inflammation. STATs are activated by tyrosine phosphorylation, predominantly JAK, leading to their phosphorylation on activating tyrosine residues, dimerization and nuclear translocation. JAKs bind specifically to intracellular domains of cytokine receptor signaling chains and catalyze the ligand-induced phosphorylation of themselves for activation (36). DN is possibly the most well-described renal disorder in which JAK/STAT activation is important. Ang II-mediated activation of JAK/STAT pathway in glomerular mesangial cells has been shown to enhance the production of transforming growth factor (TGF) $\alpha$, collagen IV and fibronectin, which is considered to contribute to the accumulation of extracellular matrix and glomerulosclerosis in DN (37). It has been reported that melatonin reduces renal inflammation and the accumulation of extracellular matrix (collagen, $\alpha$-smooth muscle actin and TGF $\alpha$ ) in chronic renal disease (38). The present study also demonstrated that, in 


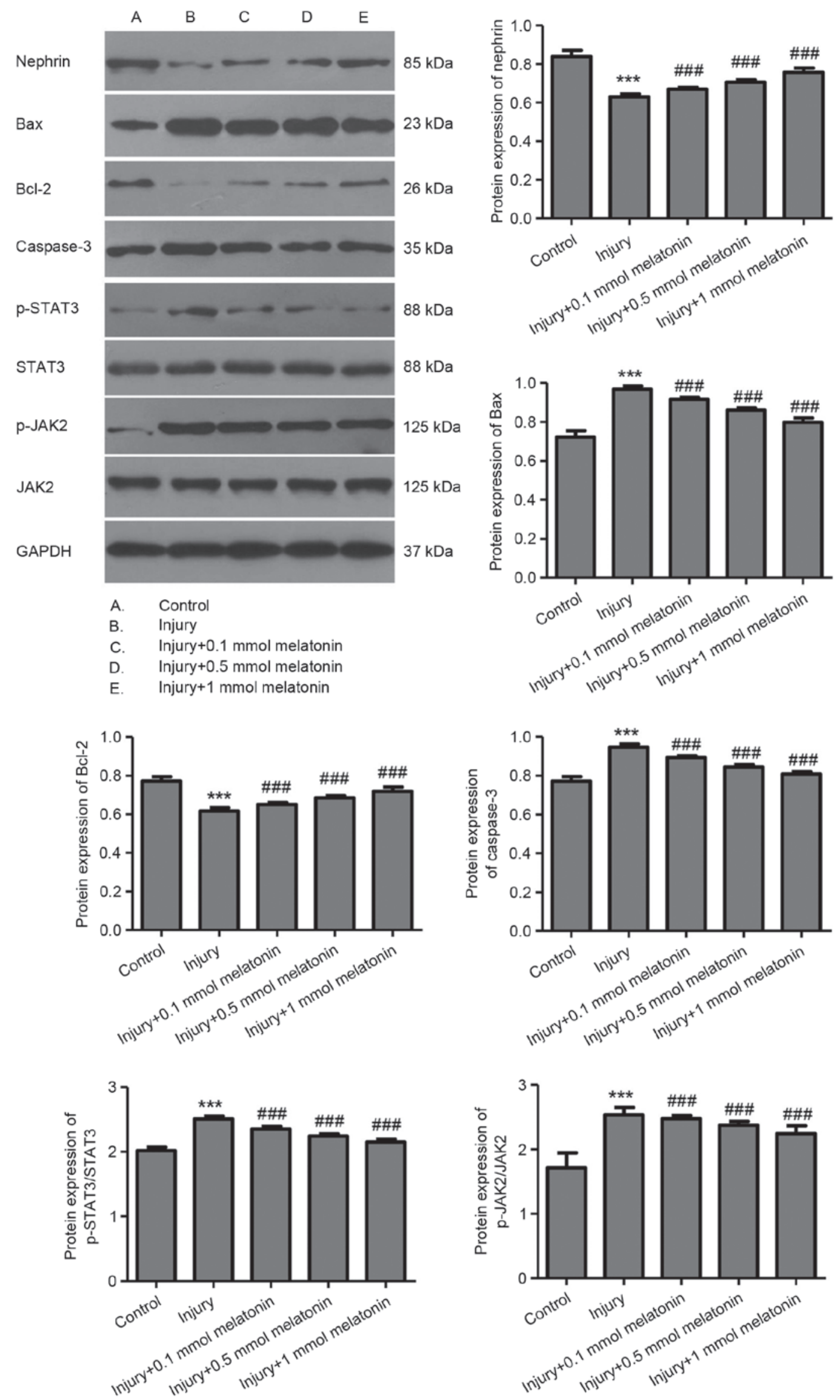

Figure 5. Western blot analysis of the relative protein levels of Nephrin, Bax, Bcl-2, Caspase-3, p-STAT3/STAT3 and p-JAK2/JAK2. Podocytes were injured by treatment with angiotensin II for $24 \mathrm{~h}$, and treated with melatonin at different concentrations. GAPDH was detected as a loading control. The molecular weight for each protein is labeled respectively. Data are expressed as the mean \pm standard deviation. ${ }^{* * *} \mathrm{P}<0.001$, injury group, vs. control group; ${ }^{\# \# \#} \mathrm{P}<0.001$, melatonin group, vs. injury group. Bcl-2, B cell lymphoma-2; Bax, Bcl-2-associated X protein; STAT3, signal transducer and activator of transcription 3; JAK2, Janus kinase 2; p-, phosphorylated. 
podocytes, the long-term administration of Ang II activated the JAK/STAT pathway through promoting the secretion of certain unknown cytokines, as reported by Abkhezr and Dryer (39). This activation of the JAK/STAT signaling pathway was inhibited by different concentrations of melatonin, possibly via interference with pro-inflammatory cytokines induced by Ang II, however, the underlying mechanism requires further investigation.

In conclusion, as a multi-functional hormone secreted by the pineal gland, the ability of melatonin to ameliorate the progression of DN was demonstrated in an Ang II-mediated podocyte-injury model in vitro. Melatonin provided protection from oxidative stress due to mitochondrial dysfunction, and inhibited apoptosis and pro-inflammatory signaling in the podocytes via repression of caspase/Bax/Bcl-2 signaling and inactivation of the JAK/STAT pathway. However, the mechanism by which melatonin is involved in this process remains to be fully elucidated, and investigations concerning the biostructure of melatonin or specific signaling pathways are required for further clinical application in DN and other renal disorders.

\section{Acknowledgements}

This study was supported by the National Natural Science Foundation of China (grant no. 81170769).

\section{References}

1. Adler S: Structure-function relationships associated with extracellular matrix alterations in diabetic glomerulopathy. J Am Soc Nephrol 5: 1165-1172, 1994.

2. Mogensen CE, Christensen CK and Vittinghus E: The stages in diabetic renal disease. With emphasis on the stage of incipient diabetic nephropathy. Diabetes 32 (Suppl 2): S64-S78, 1983.

3. Leehey DJ, Singh AK, Alavi N and Singh R: Role of angiotensin II in diabetic nephropathy. Kidney Int Suppl 77: S93-S98, 2000.

4. Lewis EJ, Hunsicker LG, Bain RP and Rohde RD: The effect of angiotensin-converting-enzyme inhibition on diabetic nephropathy. The Collaborative Study Group. N Engl J Med 329 $1456-1462,1993$.

5. Brenner BM, Cooper ME, de Zeeuw D, Keane WF, Mitch WE, Parving HH, Remuzzi G, Snapinn SM, Zhang Z and Shahinfar S; RENAAL Study Investigators: Effects of losartan on renal and cardiovascular outcomes in patients with type 2 diabetes and nephropathy. N Engl J Med 345: 861-869, 2001.

6. Gross ML, El-Shakmak A, Szábó A, Koch A, Kuhlmann A, Münter K, Ritz E and Amann K: ACE-inhibitors but not endothelin receptor blockers prevent podocyte loss in early diabetic nephropathy. Diabetologia 46: 856-868, 2003.

7. Somlo S and Mundel P: Getting a foothold in nephrotic syndrome. Nat Genet 24: 333-335, 2000 .

8. Ma L and Fogo AB: Role of angiotensin II in glomerular injury. Semin Nephrol 21: 544-553, 2001.

9. Fogo AB: Renal fibrosis and the renin-angiotensin system. Adv Nephrol Necker Hosp 31: 69-87, 2001.

10. Griendling KK and Ushio-Fukai M: Reactive oxygen species as mediators of angiotensin II signaling. Regul Pept 91: 21-27, 2000.

11. Swaminathan S and Shah SV: Novel approaches targeted toward oxidative stress for the treatment of chronic kidney disease. Curr Opin Nephrol Hypertens 17: 143-148, 2008.

12. Hakim FA and Pflueger A: Role of oxidative stress in diabetic kidney disease. Med Sci Monit 16: RA37-RA48, 2010.

13. Ha H, Yu MR and Kim KH: Melatonin and taurine reduce early glomerulopathy in diabetic rats. Free Radic Biol Med 26: 944-950, 1999.

14. Oktem F, Ozguner F, Yilmaz HR, Uz E and Dündar B: Melatonin reduces urinary excretion of $\mathrm{N}$-acetyl-beta-D-glucosaminidase, albumin and renal oxidative markers in diabetic rats. Clin Exp Pharmacol Physiol 33: 95-101, 2006.
15. Zhang H, Zhang HM, Wu LP, Tan DX, Kamat A, Li YQ, Katz MS, Abboud HE, Reiter RJ and Zhang BX: Impaired mitochondrial complex III and melatonin responsive reactive oxygen species generation in kidney mitochondria of $\mathrm{db} / \mathrm{db}$ mice. J Pineal Res 51: 338-344, 2011

16. Elbe H, Vardi N, Esrefoglu M, Ates B, Yologlu S and Taskapan C: Amelioration of streptozotocin-induced diabetic nephropathy by melatonin, quercetin, and resveratrol in rats. Hum Exp Toxicol 34: 100-113, 2015.

17. Kim EY, Alvarez-Baron CP and Dryer SE: Canonical transient receptor potential channel (TRPC) 3 and TRPC6 associate with large-conductance $\mathrm{Ca} 2+$-activated $\mathrm{K}+(\mathrm{BKCa})$ channels: Role in BKCa trafficking to the surface of cultured podocytes. Mol Pharmacol 75: 466-477, 2009.

18. Lim AKh: Diabetic nephropathy-complications and treatment Int J Nephrol Renovasc Dis 7: 361-381, 2014.

19. Mundel P and Kriz W: Structure and function of podocytes: An update. Anat Embryol (Berl) 192: 385-397, 1995.

20. Faul C, Asanuma K, Yanagida-Asanuma E, Kim K and Mundel P: Actin up: Regulation of podocyte structure and function by components of the actin cytoskeleton. Trends Cell Biol 17: 428-437, 2007.

21. Endlich N, Kress KR, Reiser J, Uttenweiler D, Kriz W, Mundel P and Endlich K: Podocytes respond to mechanical stress in vitro. J Am Soc Nephrol 12: 413-422, 2001.

22. Durvasula RV, Petermann AT, Hiromura K, Blonski M, Pippin J, Mundel P, Pichler R, Griffin S, Couser WG and Shankland SJ: Activation of a local tissue angiotensin system in podocytes by mechanical strain. Kidney Int 65: 30-39, 2004.

23. Anwar MM and Meki AR: Oxidative stress in streptozotocin-induced diabetic rats: Effects of garlic oil and melatonin Comp Biochem Physiol A Mol Integr Physiol 135: 539-547, 2003.

24. Lenz O, Elliot SJ and Stetler-Stevenson WG: Matrix metalloproteinases in renal development and disease. J Am Soc Nephrol 11: 574-581, 2000.

25. Gumustekin M, Tekmen I, Guneli E, Tugyan K, Topcu A, Ergonen AT, Ozdemir MH, Uysal N and Bediz CS: Short-term melatonin treatment improved diabetic nephropathy but did not affect hemorheological changes in diabetic rats. Pharmazie 62: 693-698, 2007.

26. Ehrenberg B, Montana V, Wei MD, Wuskell JP and Loew LM: Membrane potential can be determined in individual cells from the nernstian distribution of cationic dyes. Biophys J 53: 785-794, 1988.

27. Nicholls DG and Ward MW: Mitochondrial membrane potential and neuronal glutamate excitotoxicity: Mortality and millivolts. Trends Neurosci 23: 166-174, 2000.

28. Kanwar YS, Wada J, Sun L, Xie P, Wallner EI, Chen S, Chugh S and Danesh FR: Diabetic nephropathy: Mechanisms of renal disease progression. Exp Biol Med (Maywood) 233: 4-11, 2008.

29. Scott JA and King GL: Oxidative stress and antioxidant treatment in diabetes. Ann N Y Acad Sci 1031: 204-213, 2004.

30. Brezniceanu ML, Liu F, Wei CC, Tran S, Sachetelli S, Zhang SL, Guo DF, Filep JG, Ingelfinger JR and Chan JS: Catalase overexpression attenuates angiotensinogen expression and apoptosis in diabetic mice. Kidney Int 71: 912-923, 2007.

31. Durvasula RV and Shankland SJ: Activation of a local renin angiotensin system in podocytes by glucose. Am J Physiol Renal Physiol 294: F830-839, 2008.

32. Stitt-Cavanagh E, MacLeod L and Kennedy C: The podocyte in diabetic kidney disease. Scientific World Journal 9: 1127-1139, 2009.

33. Welsh GI and Saleem MA: Nephrin-signature molecule of the glomerular podocyte? J Pathol 220: 328-337, 2010.

34. Adams JM and Cory S: Life-or-death decisions by the Bcl-2 protein family. Trends Biochem Sci 26: 61-66, 2001.

35. Hengartner MO: The biochemistry of apoptosis. Nature 407: 770-776, 2000

36. Aaronson DS and Horvath CM: A road map for those who don't know JAK-STAT. Science 296: 1653-1655, 2002

37. Chuang PY and He JC: JAK/STAT signaling in renal diseases. Kidney Int 78: 231-234, 2010.

38. QuirozY,FerrebuzA,RomeroF, VaziriND and Rodriguez-Iturbe B: Melatonin ameliorates oxidative stress, inflammation, proteinuria and progression of renal damage in rats with renal mass reduction. Am J Physiol Renal Physiol 294: F336-F344, 2008.

39. Abkhezr M and Dryer SE: Angiotensin II and canonical transient receptor potential-6 activation stimulate release of a signal transducer and activator of transcription 3-activating factor from mouse podocytes. Mol Pharmacol 86: 150-158, 2014. 